NORTH-HOLLAND

\title{
Virtual Lines, a Deadlock-Free and Real-Time Routing Mechanism for ATM Networks
}

GERARD J. M. SMIT

PAUL J. M. HAVINGA

and

WALTER H. TIBBOEL

Department of Computer Science, University of Twente, P.O. Box 217, 7500 AE Enschede, The Netherlands

\section{ABSTRACT}

In this paper, we present a routing mechanism and buffer allocation mechanism for an ATM switching fabric. Since the fabric will be used to transfer multimedia traffic, it should provide a guaranteed throughput and a bounded latency. We focus on the design of a suitable routing mechanism that is capable of fulfilling these requirements and is free of deadlocks. We will describe two basic concepts that can be used to implement deadlock-free routing. Routing of messages is closely related to buffering. We have organized the buffers into parallel FIFO's, cach representing a virtual line. In this way, we not only have solved the problem of head of line blocking, but we can also give real-time guarantees. We will show that for local high-speed networks, it is more advantageous to have a proper flow control than to have large buffers. Although the virtual line concept can have a low buffer utilization, the transfer efficiency can be higher. The virtual line concept allows adaptive routing. The total throughput of the network can be improved by using alternative routes. Adaptive routing is attractive in networks where alternative routes are not much longer than the initial route(s). The network of the switching fabric is built up from switching elements interconnected in a Kautz, topology.

\section{INTRODUCTION}

In the past decade, we have seen a revolution in VLSI tcchnology. The transmission and switching technologies have rapidly advanced to higher spceds; it is now technically feasible to build networks with gigabit 
throughput. Ficld programmable gate arrays (FPGA) is a technology that not only allows gate arrays to be reprogrammed an unlimited number of times, but also has on-chip static memory (the X 4000 family of Xilinx). Using programmable elements gives us the opportunity to learn from experiences on prototypes and to adapt the architecture.

We are currently building a prototype network using these off-the-shelf technologies. Our goal is to build a local area network that supports multimedia applications. These applications require not just high transmission speeds, but also small end-to-end latency with little variation, a guaranteed throughput, graceful degradation under heavy workloads, and performance that is both fair and predictable. Low-latency services are necessary for voice and video transfer, process control, remote sensing, etc. The data for these services is usually worthless if it does not arrive in time. A video sequence, for instance, must be retrieved at a high and constant rate; frames retrieved too late are no longer useful and can be ignored. An additional problem is the synchronization of different media. These applications require real-time and reliable communications where certain strict deadlines must be met.

The bandwidth of many existing networks is by far not enough for distributed multimedia applications. Their throughput and latency are becoming a bottlencck in demanding real-time applications. One reason is that these networks use a single shared path for their communication. Point-to-point networks are usually organized as star-shaped networks, in which all stations are connected by dedicated links to a central switching fabric. A connection between two stations is established through the switching fabric. The main advantages of point-to-point networks are that, they offer an aggregate network bandwidth that can be much larger than the throughput of a single link, and have a high availability by allowing multiple paths. The interfaces in the stations can be simple and low cost (most of the complexities are contained within the switching fabric) and the design is relatively independent of the technology of the physical layer of the links. If there are multiple paths in the switching fabric, the connections will experience a higher availability.

In this paper ${ }^{1}$, we present, a network that can be used to transfer multimedia traffic with the above requirements.

\footnotetext{
${ }^{1}$ Some results obtained about the communication infrastructure in the Pegasus research project [5] are presented in this paper. The Pegasus project is a project of the Universities of Twente and Cambridge, supported by the European Communities Esprit Programme through BRA project 6586. It is partially supported by the Cambridge Olivetti Research Laboratory and a grant from Digital Equipment Corporation.
} 


\section{DEADLOCK-FREE ROUTING MECHANISMS}

Asynchronous transfer mode (ATM) has emerged as a leading technique for high-speed packet switching. In packet-switched networks, packets are exchanged over communication channels between nodes. In order to provide an arbitrary and fully dynamic connectivity in a static network of nodes, routing mechanisms must be implemented, which provide the propagation of data from node to node. Routing information is contained in each packet, such that it can be routed from source to destination.

A method that is very suitable for ATM packet switching is wormhole routing. With this method, all packets of a message are routed on the same path from source to destination. During connection setup, a path from source to destination is claimed for that message. Wormhole routing prevents the ordering of packets from being altered.

Depending on the network topology to which it is applied, routing may be subject to deadlock problems. Deadlocks occur when we can identify a cyclic buffer waiting graph, for example, when none of the packets in the network can advance towards its location, because the necessary queue for that packet on the next node is already full.

In this section, we will describe two basic concepts for deadlock-free routing. We will show that a virtual line concept suits very well our demands of a multimedia network, and that it has a low complexity. The main design issue is how to organize the buffers.

\subsection{MAIN CONCEPTS}

There exist a number of deadlock-free routing algorithms. Most algorithms are based on the use of static buffer graphs whose structure ensures that deadlock situations cannot occur. Taking cycle-free buffer graphs will be sufficient to prevent the occurrence of any circularity in resource (i.e., buffer) requests.

A basic concept in deadlock prevention is to guarantee a path in the network. If every message injected in the network follows one of its guaranteed paths, deadlock cannot occur since there are no directed loops in the buffer graph. We will describe two main concepts.

- Class climbing is a general concept which is often used. When a process (e.g., a task that sends a message from source to destination) has rank $r$, that is, it has already obtained resources of class $r$, it may only request resources of classes higher than $r$. Figure 1 gives an example. There must be sufficient classes of resources (buffers) to satisfy the rank in the network. The number of buffers required per node depends on the maximum number of hops possible in the network, which in its turn depends on the network topology and on 


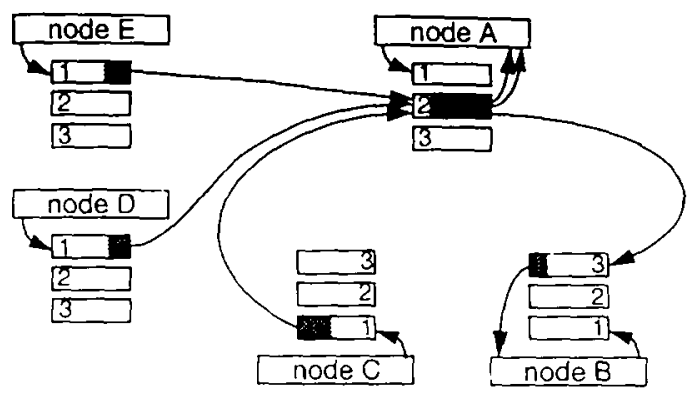

Fig. 1. Class climbing concept.

the routing properties. Several schemes have been proposed in the literature for constructing buffer graphs and to assign ranks: virtual graphs, valley counting, hops-so-far (hop counting), etc. 'These approaches can restrict the routing of packets: they reduce the number of paths that a packet may take [1]. The length of the path can be longer than the shortest path; such a route implies more latency.

- Another approach is to use a connection oriented network service. The basic concept, is to guarantee a path in the network. If every message injected in the network follows one of its guaranteed paths, deadlock cannot occur since there are no directed loops in the buffer graph. The method of nosy worms [10] can be used for this approach. During the connection phase, at every node needed to form the virtual communication channel, enough buffers are reserved to support the connection. 'These buffers will only be released when the connection is terminated. Messages start off by being buffered at their sending node. Each message attempts to establish a connection with the destination node by forming an unbroken path across intermittent nodes. If a block or a failure is encountered along the route, the message gives up by recoiling back to the sender, thus avoiding deadlock. If there are alternative routes, other routes can be tried. Once a connection is made, the entire message is transferred from source to destination, where it is buffered again before it is off-loaded to the receiving system. In fact, this is a kind of circuit switching. The main disadvantage of this approach is that it has a low buffer utilization. Circuit switching is often used in combination with virtual channels.

\subsection{VIRTUAL LINES}

An important decision to make is how to organize the buffers. We assume that all buffers are organized as FIFO's. A dynamic buffer structure, which 


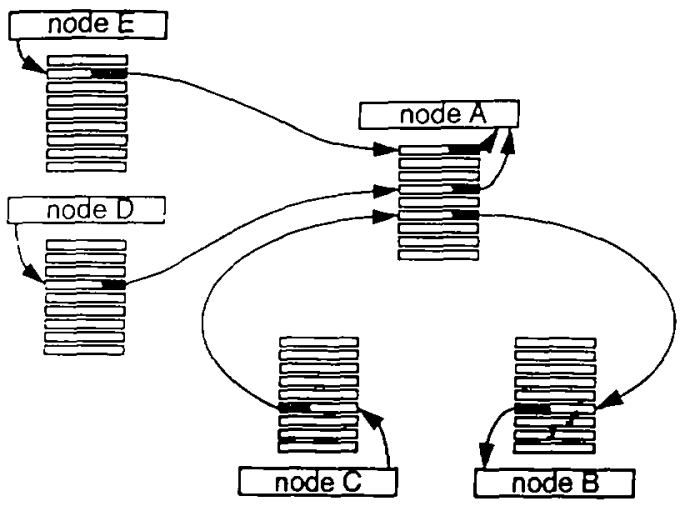

Fig. 2. Virtual line concept.

is commonly used in software solutions, seems not easy to implement in hardware. A more or less static buffer structure is easier to implement. One important problem associated with buffering is the severe throughput degradation due to head of line (HOL) blocking. Whenever a FIFO is filled with data that cannot be forwarded, all the data which enter later will be blocked also.

In this context, we have to make a trade-off between performance and buffer utilization. When the buffers are organized as (one or more) large FIFO's, the efficiency of these buffers can be high, since all buffer space can be used. From a performance point of view, a better approach is to use parallel buffers.

A switch may organize the input buffers by associating them with virtual lines, i.e., a connection between sender and receiver. Virtual networks, implementing a number of virtual lines on one physical link, was first introduced as a technique to avoid deadlocks in networks. Virtual lines decouple allocation of buffers from allocated lines by providing multiple buffers for each channel in the network. A blocked message, even one that extends through several links (e.g., in case of wormhole routing), blocks only one virtual line and can be overtaken by messages in other virtual lines. Figure 2 shows as an example the line buffers of five nodes.

Dally [2] showed that virtual networks increase the connectivity of networks and have performance advantages as well. The disadvantage is that buffer utilization is low. However, as the cost of memories is fairly low, it seems more important to realize an efficient data transfer than to have a high buffer utilization. 
Since the total available buffer space is divided into many small buffers, we need to have an efficient flow control mechanism to prevent buffer overflow. In ATM, flow control has becn omitted. Bandwidth is allocated in such a way that statistically the amount of buffering is sufficicnt most of the time. Cells that arrive at an input port with full buffers are discarded; evidently not a subtle method, but necessary to avoid deadlock. This lack of flow control has been adopted for simplicity reasons; it is very difficult to implement a flow control over high-speed links bridging large distances. In a local network environment, however, such as within a switching fabric, it is possible to perform a proper flow control. The main advantage of an efficient flow control is that there is no longer need for huge buffers that are capable of dealing with the high-speed networks. Moreover, evcn with large buffers, it is not possible to prevent loss of cells. With speeds of $100 \mathrm{Mb}$ per second and more, even large memories will be filled quickly. Adding the number of buffers (lines) is a more efficient use of storage than adding depth to a buffer.

\subsection{CONCLUSION}

The most important difference between the two concepts is the buffering. The class climbing concept uses large buffers that can be used by all connections, whereas the virtual line concept uses small buffers in which each buffer belongs to one connection. Other properties are:

- The number of simultaneous connections using virtual lines is restricted to the number of buffers. The class climbing concept, however, supports an unlimited number of connections.

- 'There is an automatic workload distribution duc to the nosy worms protocol. If the shortest route contains a contented node, the algorithm will automatically select another (node disjoint) route.

- Both methods are deadlock-free. When all virtual lines of a link from a route from source to destination are already claimed, the nosy worms protocol bounces back to the source, thereby avoiding deadlocks. The source will then try another route.

- The virtual lines have a bounded latency. The class climbing concept cannot give any bandwidth guarantecs.

- The buffer utilization of virtual lines is poor. The memory is split up into many lines. The lines can only use their allocated memory, even though other lines have buffers available. However, the virtual line concept has not the intention to solve local bandwidth problems of a high-speed network with large memory storage, because even large memories will be filled quickly. The buffer utilization of class climbing is high, but suffers from HOL blocking. 


\section{THE RATTLESNAKE PORT CONTROLLER}

In this section, we will give some details about the prototype switch called Rattlesnake. 'The switch consists of a number of switching elements interconnected via bidirectional links. The switching elcments are configured in a Kautz network topology [4] because of its valuable properties. Particularly, Kautz graphs have a small diameter, a fixed and a small degree [3].

Each switching element is connected to a port controller (called snake control). The external links of the port controller are point-to-point links $(>100 \mathrm{Mbit} / \mathrm{s})$ using the A'IM transfer mode. These links are connected to a (work)station or a server. The port controller has local memory in which the AI'M cells coming from and going to the serial link are buffered.

The communication architecture should provide a guaranteed throughput and a bounded latency. Therefore it must be able to establish realtime connections. We realize this with bandwidth reservation using virtual channels. A connection between two arbitrary stations is made via two or more switching elements in the switching fabric. A message generated by a source station travels through these switching elements to reach a destination station. The switching elements forward messages from an input link to an output link, as directed by the destination address in each message header.

\subsection{RATTIESNAKE ROUTING AND BUFFERING}

The port controller is connected to a switching clement (SE) [6] that supports real-time connections with other SEs. A SE has three input links and three output links; cach link contains 16 small buffers. These buffers are used to implement 16 virtual channels on one physical link. Note that the term "channel" is directly related to the physical connection between SEs, whereas the tcrm "line" is related to the connection bctween port controllers. In a switching element, only a few flits are buffered. A fit is the smallest unit of data and is in our case 32 bits. When establishing a connection from source to destination, each SE assigns an input channel and an output channel for this circuit. To find a connection from source $\mathrm{SE}$ to destination $\mathrm{SE}$, a wormhole routing mechanism is used. Wormhole routing operates by advancing the head of a packet directly from incoming to outgoing channels. As soon as a node examines the header flit of a message, it selects the next channcl on the route and begins forwarding flits down that channel. The path of a logical connection is set for the duration of a conncction. 'This means that messages of a certain conncction will always follow the same path through the switch. 
In the design of the snake controller, we also use a kind of wormhole routing, called virtual line routing [8]. In the snake controller, packets (i.e., ATM cells) are buffered. The buffer space is located in off-chip memory. This memory is divided into a number of buffer queues, called virtual lines. The snake controller uses the virtual channels of the switching clement (SE) to make real-time connections to other snake controllers (SC's). The virtual lines are put on top of the virtual channels of a SE. So on each virtual channel between SE's, there can be several virtual lines from a SC. The circuit-switched connections between SC's may be to the neighbor SE, but it may also traverse several SE's before reaching the next SC. In this way, it is possible to create a highway between SC's with a high mutual communication load.

A path from source of destination is established by reserving and linking the virtual lines in each intermediate $\mathrm{SC}$ on the path. The packets are transferred on a hop-by-hop basis from SC to SC until the destination is reached. Each cell is first received by a SC in its full length at each intermediate SC between source and destination, and then it is transmitted to the next, SC. If a failure or a blocking condition is encountered along the route, the setup message gives up by recoiling back to the sender, thus avoiding deadlock. Another (node disjoint) route can be tricd. ${ }^{2}$ 'This leads to an adaptive routing mechanism that routes around congestions and faults. Adaptive routing is attractive in networks where alternative routes are not much longer than the first route(s). The averaged hop lengths in our prototype network of 108 nodes in a Kautz topology of the first, second, and third route are, respectively, $3.5,4.9$, and 5.2 hops [8].

By this combination of circuit switching and packet switching, we have created a hybrid transfer mode. We have combined the real-time circuitswitched network between SE's with the flexibility of packet switching betwcen SC's. Applications gencrating hard real-time data are able to reserve a circuit-switched connection from source to destination. Less demanding applications, such as file transfers, may use the packet-switched component of the transfer mode. The hybrid switching mode appears to be particularly attractive for networks that have to support dissimilar traffic of both continuous and bursty types generated by heterogeneous users, such as networks for multimedia traffic. A circuit-switched connection can be used to satisfy the stringent real-time and latency constraints for continuous traffic, and the packet connection can be used for non-real-time traffic.

\footnotetext{
${ }^{2}$ The probability of a connection message reaching its destination depends primarily on the chances of finding an unbroken chain of channels. [7] gives simulation results showing that these chances are improved by using a low-diameter network (e.g., Kautz), using alternative routes, and by using virtual channels.
} 


\subsection{SNAKE CONTROL ARCHITECTURE}

Figure 3 shows the global view of a node. Each node contains a switch, a snake control, and two memories. One memory is the line momory, where data of the network can be stored. The other memory is the table memory, which is used for administration storage.

The communication between the host and its nctwork node is based on ATM cells. When a host transmits an ATM cell to the node, the port. controller will store the cell in the virtual line queue that was reserved for the ATM virtual channel (indicated by the VCI in the header of the cell). A virtual line connection between port controllers is put on top of the virtual channels of a SE. Therefore a virtual line identifier (VLI) contains three elements: outgoing link number, virtual channel number, and the line number on the channel.

The snake controller splits up ATM cells coming from the stations into flits and transmits them to its switching element. A fit consists in our case of 32 bits data and 8 bits identification. The identification consists of a 4-bit virtual channel number and 4 bits type. In addition to this data path, there is a 1-bit reverse status path that can be uscd to implement a flow control mechanism. 'The flow control can prevent loss of cells due to buffer overflow not just within the SE, but also within the SCs.

Figure 4 shows the internal organization of a snake controller. It has 640 parallel line queues. These queues are used to implement the virtual lines. When establishing a connection from source to destination, each SC assigns a line queue for this circuit. The routing information (i.e., the selected outlink, the virtual channel to be used, and the virtual line) is

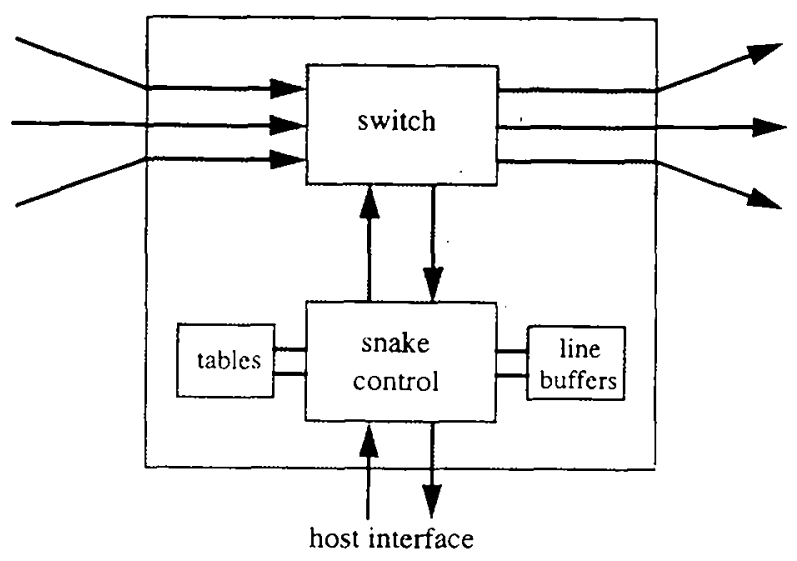

Fig. 3. Structure of a network node. 


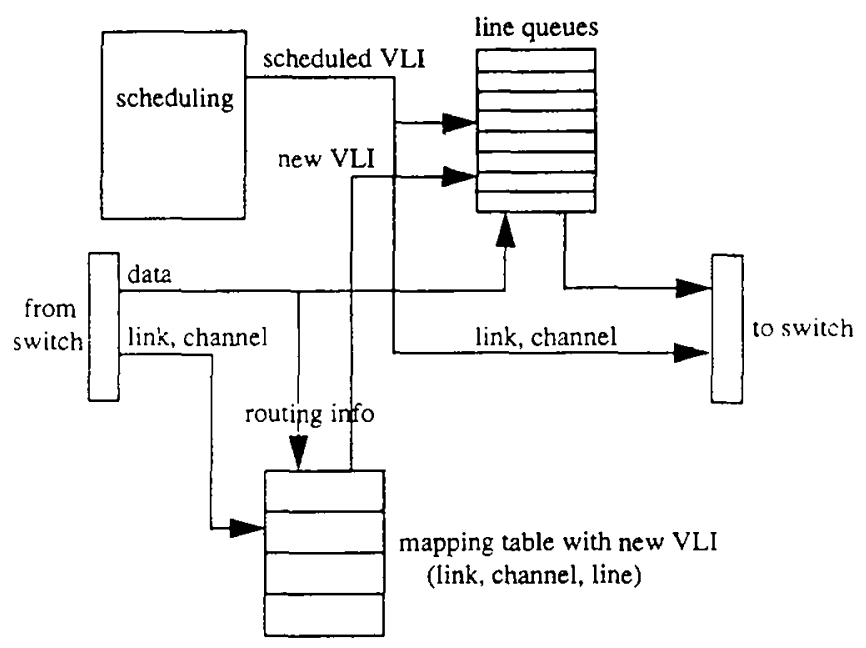

Fig. 4. Structure of the port controller.

stored in a mapping table. Each entry in the table contains the new virtual channel number, the selected outlink, and the line on the virtual channel. 'This information is used for the connection with the next SE. Each entry in the mapping table has only local significance and identifies the local virtual line translation.

The receiving snake control receives the flit data and the corresponding virtual channel and the inlink number. This information is used to index the table to generate the now virtual line. 'This line is used to select the required line queues.

\subsection{SCHEDULING}

The scheduler of the sending snake control has the most important and difficult task: it must select a flit of an ATM cell that must be transmitted to the next SE.

On one virtual channel, the SC will transmit whole ATM cells only. A complete coll is transmitted, interleaved with cells of other channels, before the next line of the same channel will be scheduled. The current implementation of the scheduler uses a simple round robin mechanism to select a line that contains an ATM cell. 'The scheduler is the most critical part of the SC design with respect to the implementation limitations in time and space. In the current implementation, it must choose from 80 virtual channels, and within cach virtual channel it must schedule 8 lines. The scheduling problem is split up into two layers. First, a virtual channel 


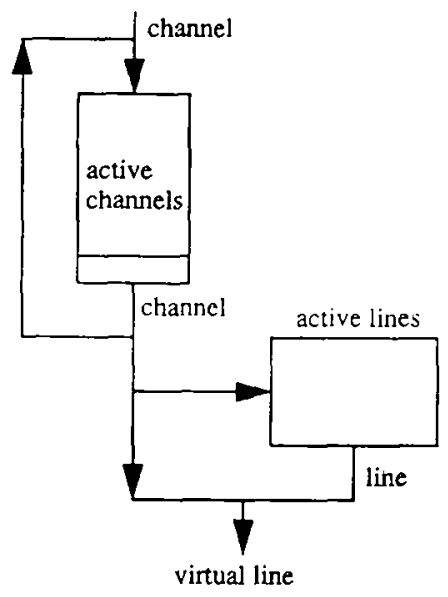

Fig. 5. Scheduling.

for a link in scheduled, and second, a line of that channel is scheduled (see Figure 5).

- The channel scheduler is implemented with a FIFO of 80 deep. The FIFO contains channels that have an ATM cell in a line queue. Every time an ATM packet is received from either the host or from another port controller, the channel will be put on top of the FIFO. Such a FIFO can be implemented with on-chip momory.

- The line scheduler uses a bit array to administrate the status of the lines, indicating which lines are active of that channcl. This status, together with the current line, gives the next active line. This line is stored in a lookup table containing the currently active lines of a channel.

The scheduler will select one line and read one flit from the queuc. This line will be offered to the SE or to the host. When the flit is destined for the SE and it has not yet transmitted the end of the ATM cell, the line will be put on top of the FIFO again. A scheduled line that is destined for the host will transmit a whole A'T'M cell.

\subsection{CONNECTION SETUP}

The connection setup procedure requires connections on two layers. First, it requires the circuit-switched conncction bctween SE's. 'These connections are used as point-to-point connections betwcen SC's. Such 
a connection in general reaches the neighbor SE only, but it may pass several SE's. The circuit is used to transfer the packets of various virtual lines. Hard real-time connections can have their own private circuit from source to destination. The circuit-switched connections are static: once they are set up, they remain intact for a long period. 'T'he wormhole routing mechanism is used to reserve buffers in a SE.

The second connection layer is a packet-switched end-to-end connection betwecn the source and destination of the message. The packets are transmitted on a hop-by-hop basis through one or several SC's bcfore reaching the destination. The packet-switched connections are more dynamic: the connection will be released after a message transfer is completed. In this way, the line buffers are released and can be used for other connections. The packet-switched connections also use the wormhole routing mechanism. This requires that each node of the path has a free line. Only when all the nodes of the path can reserve a virtual line can a connection be established. When a node has no frec line, this node must inform all the preceding nodes which did reserve a virtual line, so that they can cancel the reservation. Only the last node of the connection can return a status indicating that the connection is established.

To find a path from source to destination, we take advantage of the self-routing property of Kautz networks. The host which will set up a connection must generate a connection setup request cell. This cell contains the route to be followed, called the routing tag. The consecutive digits of the routing tag are interpreted stage-by-stage. If this connection setup effort fails because one of the links of the path is already completcly reserved, the host will try an alternative route.

The path of a logical connection is set for the duration of a connection. This means that messages of a certain connection will always follow the same path through the switching fabric until it receives a release cell. The $\mathrm{SC}$ will transmit the release cell to the other SC's downstream.

\subsection{IMPLEMENTATION}

The architecture of the port controller uses several memories. These memories are uscd to implement the lookup tables, the scheduling FIFO's, the line queues, and some local administration. Most of the basic elements of the SC operate concurrently to meet the timing requirements needed to handle the high-speed bandwidth rates. The transmission of a coll to the external link operates simultaneously with the reception of a cell from the link. Likewise, the transmission and reception of fits to and from the SE also occur simultaneously. Simultaneous access to tables 
and FIFO's is required to achieve these operations. One shared memory would create an enormous bottleneck that can only be solved using a wide data path, or very high-speed memorics. A better way is to use separate memories. We make extensive use of the distributed mernories inside the Xilinx X4000 FPGA [11]. Due to the small capacity of these memories, they can only be used to implement several small tables. The large tables and the line queues are implemented using two external momories.

The prototype printed circuit board with one network node contains two Xilinx PG4010 FPGA's to implement the switching element and the snake control, and two memories of 2 Mbytes connected to the snake control. A switch has three input and threc output links each with nine wires. These wires can be sciected unidirectional or bidirectional. The interface with the node computers is provided using TAXI links. ${ }^{3}$

This prototype board allows us to do experiments with and performance measurements of sevcral transmission modes, bandwidth reservation, scheduling, routing for non-real-time traffic, link protocols, multicast, etc. We use VHDL as a design tool and a VHDL synthesizer from VIEWlogic [9] to generate the configuration code for the Xilinx chips.

\section{CONCLUSION}

In this paper, we have presented the architecture of a network suitable for hard real-time multimedia applications. This ATM network uses a modified nosy worm protocol for setting up connections. Once a connection is established, it guarantees a bounded latency. We make use of virtual lines to improve the performance and to avoid communication deadlocks.

The buffering of the messages is main topic. We have organized the buffers as parallel FIFO's, each representing virtual line. In this way, we have not only solved the problem of HOL blocking, but we can also give real-time guarantees. We have shown that for local high-speed networks, it is more advantageous to have a proper flow control than to have large buffers. Although the virtual line concept can have a low buffer utilization, the transfer efficiency can be higher.

The virtual line concept allows adaptive routing. The total throughput of the network can be improved by using alternative routes. Adaptive routing is attractive in networks where alternative routes are not much longer than the first route(s).

A prototype of the switching fabric is implemented with standard FPGA's. We use two external memories and several on-chip memories

\footnotetext{
${ }^{3} \mathrm{We}$ use the Fairile transmission board designed by the University of Cambridge.
} 
to implement the various tables and FIFO's. This allows us to access the memories concurrently and meet the timing requircments. The design of the switching fabric with FPGA's makes it possiblc to experiment with switching mode, routing strategy, and scheduling policy in a multimedia environment.

\section{REFERENCES}

1. J. M. Adamo, Minimal, adaptive and deadlock-free routing for multiprocessors, Laboratoire de LIP-IM AG, Ecole normale superieure de Lyon, France, June 1991.

2. W. J. Dally; Virtual-channel flow control, IEEF Transactions on Parallel and Distributed Systems 3(2):194-205 (1992)

3. M. Imase, T. Soncoka, and K. Okada, A fault-tolerant processor interconnection network (original in Japanese); translated in Systems and Computers in Japan $17(8): 21-30(1986)$.

4. W. H. Kautz, Bounds on directed $(d, k)$ graphs. Theory of cellular logic networks and machines, AFCRI,-68-0668 Final report, pp. 20-28, 1968.

5. I. M. Leslie, J). Mc $\Lambda$ uley; and S. J. Mullender, Pegasus--Operating system support for distributed multimedia systems. ACM SIGOPS Operating System Revieu; 27(1):69-78 (1993).

6. G. J. M. Smit and P. J. M. Havinga, The architecture of Rattlesnake: A real-time multimodia network, Proceedings 12th IFIP World Congress, September 1992, pp. $578-584$.

7. G. J. M. Smit and P. J. M. Havinga, Performance analysis of routing algorithms for the Rattlesnake network, Proceedings MASCOTS '93, January 1993, pp. 155-160.

8. W. H. Tibboel, Virtual lines, a deadlock free and real-time buffer allocation mechatnism for an ATM-nctwork, MS thesis, Department of Computer Science, University of Twente, July 1993.

9. VHDL-Designer User's Guide, VIFW logic Systems Inc., April 1990.

10. D. Whobrey, A communications chip for multiprocessors, Proc. CONPAR 88, 1988 . pp. 464-473.

11. The Programmable Gate Array Data Book, Xilinx Inc., 1991.

Received 1 May 1993; revised 29 October 1994 